AperTO - Archivio Istituzionale Open Access dell'Università di Torino

\title{
Conjugation of amino-bioactive glasses with 5-aminofluorescein as probe molecule for the development of $\mathrm{pH}$ sensitive stimuli-responsive
}

This is the author's manuscript

Original Citation:

Availability:

This version is available http://hdl.handle.net/2318/143644

since 2015-12-30T18:59:30Z

Published version:

DOI:10.1007/s10856-014-5206-4

Terms of use:

Open Access

Anyone can freely access the full text of works made available as "Open Access". Works made available under a Creative Commons license can be used according to the terms and conditions of said license. Use of all other works requires consent of the right holder (author or publisher) if not exempted from copyright protection by the applicable law. 
Conjugation of amino-bioactive glasses with organic molecules 5aminofluorescein as probe molecule for the development of $\mathrm{pH}$ sensitive stimuliresponsive biomaterials

Valentina Aina ${ }^{\mathrm{a}, *}$, Gianluca Malavasi ${ }^{\mathrm{b}}$, Claudio Magistris ${ }^{\mathrm{a}}$, Giuseppina Cerrato ${ }^{\mathrm{a}}$, Gianmario Martra $^{\mathrm{a}}$, Guido Viscardi ${ }^{\mathrm{a}}$, Ledi Menabue ${ }^{\mathrm{b}}$, Gigliola Lusvardi ${ }^{\mathrm{b}}$

${ }^{\mathrm{a}}$ Department of Chemistry, Centre of Excellence NIS (Nanostructured Interfaces and Surfaces); INSTM (Italian National Consortium for Materials Science and Technology), University of Torino, Via P. Giuria 7, 10125 Torino, Italy.

${ }^{\mathrm{b}}$ Department of Chemical and Geological Science, University of Modena and Reggio Emilia, Via Campi 183, 41125 Modena, Italy.

*Corresponding author:

Valentina Aina

Department of Chemistry, University of Torino

Via P. Giuria 7. 10125 Torino, Italy

e-mail: valentina.aina@unito.it

phone: $\underline{00390116707533}$

fax: $\underline{00390116707855}$ 


\begin{abstract}
Bioceramics, such as silica-based glasses, are widely used in bone and teeth restoration. Nowadays, the association between nanotechnology and pharmacology is one of the most promising research fields in cancer therapy. The advanced processing methods and new chemical strategies allow the incorporation of drugs within them or on their functionalized surfaces. Bioceramics can act as local drug delivery systems to treat bone and teeth deseases.

The present paper reports data related to the development of a $\mathrm{pH}$-stimuli responsive bioactive glass. The glass conjugation with 5-aminofluorescein (5-AF), through a $\mathrm{pH}$-sensitive organic spacer, allows to produce a $\mathrm{pH}$-responsive bioactive biomaterial: when it is exposed to specific $\mathrm{pH}$ changes, it can favour the release of 5-AF directly at the target site.

5-aminofluorescein has been chosen as a simple, low cost, non toxic models to simulate doxorubicin an anticancer drug. As doxorubicin, 5-AF contains an amino group in its structure in order to form an amide bond with the carboxylic functionalities of the glass. Raman spectroscopy and thermal analysis confirm the glass conjugation of 5-AF by means of an amide bond; the amount of 5-AF loaded was very high $(\approx 65$ and $44 \mathrm{wt} \%)$. The release tests at two different $\mathrm{pH}(4.2$ and 7.4) show that the amount of released 5-AF is higher at acid $\mathrm{pH}$ with respect to physiological one. This preliminary datum evidenced that a $\mathrm{pH}$-sensitive drug delivery system has been developed. The low amount of 5 -AF released $(2 \%<1 \mathrm{wt} \%$. of the total $5-\mathrm{AF})$ is due to the very low solubility of 5-AF in aqueous medium. This disadvantage, may be overcome in a dynamic environment (physiological conditions), where it is possible to obtain a drug release system ensuring an effective therapeutic dose for long times and, at the same time, avoiding the drug toxicity.
\end{abstract}

Keywords: Bioceramics, Controlled drug delivery, pH-Responsive bioactive glass; Covalent surface functionalization; 5-Aminofluorescein; Maleic anhydride. 


\section{Introduction}

Bioceramics, such as calcium phosphates, cements and silica-based glasses, are widely used as components of implants for bone and teeth restoration.[1] Nowadays, the advanced processing methods and new chemical strategies allow the incorporation of drugs within them or on their functionalized surfaces. In this regard, bioceramics can act as local drug delivery systems to treat bone defects, infections, tumours, and osteoporotic fractures. The synergy of the bioactive behaviour of bioceramics together with their capability for local drug delivery is an outstanding perspective for bone therapy purposes.[2]

Specific long-term situations could require the promotion or retardation of drug release, depending on the disease evolution. For this purpose, implantable systems that are able to respond to external stimuli magnetic fields or internal $\mathrm{pH}$ changes are at the forefront of research..[3]

Between bioceramics there are melted silica-based bioactive glasses first prepared in 1969 by Hench et al.[4] From a biological and chemical point of view, they exhibit many of the properties associated with an ideal material for grafting and scaffolding. In the early 1990s bioactive glasses were prepared by the sol-gel process for the first time.[5] Their bio-functionalization provides added value to the implant, since the graft not only fills and repairs the defect, but also acts as a drug delivery system, which can supply osteoregenerative agents locally.[6]

The possible causes for a bone defect are numerous, but most of the studies involving bioceramicdrug associations have addressed one of the following therapies: $i$ ) bone regeneration in critical defects, ii) bone infection treatments, iii) osteoporotic fracture consolidation and $i v$ ) bone tumour treatments.[2,7]

Concerning tumour treatments, implantation of bioceramic bone grafts combined with specific local cancer treatments is an excellent alternative way to restore large bone defects that occur after tumour extirpation or partial bone resection resulting in tumour inhibition with low levels of systemic toxicity. Most current therapies for cancer treatment based on systemic administration of conventional cytotoxic agents cause severe side effects in the patient and are of limited effectiveness. Many studies[8-10] could be attributed to a lack of specificity of the antitumor drugs that have been developed so far, which cause collateral damage to healthy cells. To resolve these problems, one of the strategies being investigated is to design specific drug delivery systems that can target and carry on effective dose of drug molecules to cancerous cells or tissues. The success of this strategy depends on the ability to design and synthesize a biocompatible nanocarrier suitable for transferring high drug loadings, avoiding premature release of it before reaching its destination.[11-14] 
Stimuli-responsive DDSs, also denoted smart DDSs, are designed to avoid the premature release of drug molecules before the target cell or tissue is reached.[15] Changes in the $\mathrm{pH}$ of an environment is an attractive release trigger because some human tissues under pathological conditions (tumours, inflammation, etc.), as well as endosomal cell compartments, have a more acidic $\mathrm{pH}$ than human plasma and/or healthy tissues.[2,3,16]

To this purpose, a bioactive glass with a covalent $\mathrm{pH}$ sensitive functionalization has been developed. Hydrazones and the amides of unsaturated anhydrides are among the most frequently used $\mathrm{pH}$-sensitive spacers in drug delivery.[17]

The method of surface modification of glasses is crucial for the subsequent applications as $\mathrm{pH}$ responsive nanodevices. $\alpha, \beta$-Unsaturated dicarboxylic acid anhydrides were first reported as $\mathrm{pH}$ sensitive spacers by Shen and Ryser in 1981.[18] The amino group of daunorubicin was functionalized with maleic and cis-aconitic anhydrides, and the derivatives were conjugated to aminoethyl polyacrylamide beads through their carboxylic groups. The cis-aconityl linkage proved to be $\mathrm{pH}$-sensitive, with a maximum release of $66 \%$ of bound daunorubicin after $6 \mathrm{~h}$ at $\mathrm{pH} 4$. At $\mathrm{pH}$ 7 , the linker proved very stable, and no daunorubicin was detected even after 4 days. Cytotoxicity tests on WEHI-5 cells confirmed this result, showing up to $90 \%$ cell growth inhibition after $18 \mathrm{~h}$ at $\mathrm{pH} 5$, compared to a total lack of inhibition at $\mathrm{pH}$ 7. The maleic anhydride derivative, on the other hand, showed no daunorubicin release nor cytotoxicity, even after incubation in acidic medium. The authors concluded that in the intermediate cis-aconityl daunorubicin, the $\gamma$ carboxyl reacts with the amino groups on the carrier; during the release, the hydrolysis of the amide bond undergoes intramolecular catalysis by the $\beta$ carboxyl; on the other hand, the maleyl derivative, lacking the free carboxyl function, doesn't work. Following these results, a considerable amount of literature has flourished over the years; the studies are related to cis-aconitic anhydride as $\mathrm{pH}$-sensitive spacer $[19,20]$ for the drug delivery of doxorubicin, conjugated with polyamidoamines,[17,21,22] with chitosan[23,24] or modified polyethylene glycol.[25]

Only very recently, Zhang et al.[26] used maleic anhydride to conjugate doxorubicin to a folatedecorated pullulan. Pullulan was first functionalized with maleic anhydride, and then, after activation of the maleamic acid, doxorubicin was conjugated. Total amount of bound doxorubicin was determined by fluorescence spectrophotometry, and the conjugate was characterized by NMR spectroscopy. Release tests showed a release of $60 \%$ of total bound doxorubicin at pH 5.0 which is comparable with the results obtained with cis-aconitic anhydride. Cytotoxicity tests on human breast cancer cells confirmed the release results. Compared to Shen and Ryser's earlier work,[18] 
this study shed a new light on maleic anhydride as a pH-sensitive spacer, and inspired our work on the functionalization of bioactive glasses.

In this preliminary study, 5-aminofluorescein (5-AF), a derivative of fluorescein containing a primary amino group, has been used as model molecule to simulate a drug. This dye is characterized by low cost and low toxicity, and thanks to its high molar absorptivity (77,000 L $\left.\mathrm{mol}^{-1} \mathrm{~cm}^{-1}\right)$ [27] it can easily be detected by means of absorption and emission spectroscopies (absorption at $494 \mathrm{~nm}$ and emission at $519 \mathrm{~nm}$ ). It can be also used to monitor the localization of these biologically active molecules in living cells and provide a useful tool for linking biochemical investigation with optical visualization methods.[28-31] The combination of 5-AF and maleic anhydride with bioactive glasses has generated a large range of novel hybrid materials tailored to applications in localized drug delivery systems for bone diseases.

In the present paper a covalent glasses functionalization by means of two different routes has been carried out. Through these methods a $\mathrm{pH}$-sensitive bioactive glass was realized by covalently immobilizing 5-AF molecules onto the surface of the bioactive glass with maleic anhydride as linker molecule. Indeed, the efficiency of the functionalization has been verified by means of different physico-chemical characterization techniques. To verify the behaviour of the materials after the covalent functionalization/conjugation procedure, delivery tests in simulated body solution at different $\mathrm{pH}$ have been performed showing a $\mathrm{pH}$-sensitive drug delivery behaviour. 


\section{Materials}

3-Aminopropyltriethoxysilane (APTES) containing glass. In order to obtain the sol-gel aminopropyl bioactive glass the conventional $\mathrm{SiO}_{2}$ source (tetraethyl orthosilicate, TEOS) was partially substituted with 3-aminopropyltriethoxysilane (APTES). Triethylphosphate (TEP) and calcium nitrate tetrahydrate $\left(\mathrm{Ca}\left(\mathrm{NO}_{3}\right)_{2} \cdot 4 \mathrm{H}_{2} \mathrm{O}\right)$ have been used as $\mathrm{P}_{2} \mathrm{O}_{5}$ and $\mathrm{CaO}$ sources, respectively. The glass theoretical composition is $80 \% \mathrm{SiO}_{2}, 15 \% \mathrm{CaO}$ and $5 \% \mathrm{P}_{2} \mathrm{O}_{5}$ (\%mol); the percentage of APTES used as precursor of $\mathrm{SiO}_{2}$ is $25 \%$ mol. A water solution was prepared dissolving specific amounts $2.61 \mathrm{~g}$ of $\mathrm{Ca}\left(\mathrm{NO}_{3}\right)_{2} \cdot 4 \mathrm{H}_{2} \mathrm{O}$ in a mixture of $0.2 \mathrm{ml} \mathrm{HCl}(37 \mathrm{wt} \%$ \% w/w $)$ and $5.0 \mathrm{ml}$ of $\mathrm{H}_{2} \mathrm{O}$. This aqueous solution was added to $40 \mathrm{ml}$ of ethanol where $10.0 \mathrm{ml}$ of TEOS and $3.5 \mathrm{ml}$ of APTES were mixed; $1.3 \mathrm{ml}$ of TEP had been previously added to each APTES/TEOS mixtures (each addition every 3 hours), in order to obtain the stoichiometric compositions. The solutions were then stirred for 1 hour and cast in Teflon ${ }^{\circledR}$ containers, that were hermetically closed and kept 1 day at room temperature for gelation. Gels were then aged 1 day at $333 \mathrm{~K}$. In order to remove the nitrates eventually still present in the sample the gel has been washed with a $0.1 \mathrm{M} \mathrm{Ca}^{10} \mathrm{z}^{-} \mathrm{Ca}(\mathrm{OH})_{2}$ solution for 1 hour under magnetic stirring.

The aged systems were treated at $423 \mathrm{~K}$ for 3 hours in an open $\mathrm{Al}_{2} \mathrm{O}_{3}$ crucible (heating ramp: $2^{\circ} \mathrm{C} / \mathrm{min}$ ); the latter temperature value has been chosen on the basis of the thermogravimetric analyses performed on the APTES containing samples. This is the highest calcination temperature to be used in order to avoid the decomposition of the $-\mathrm{CH}_{2}-\mathrm{CH}_{2}-\mathrm{CH}_{2}-\mathrm{NH}_{2}$ chains of APTES molecules inserted during the synthesis. Finally the obtained powders were grounded in an agate mortar to $\varnothing<500 \mu \mathrm{m}$. This starting glass will be named APTES-SG. APTS25SG423.

\section{Methods}

\section{Functionalization and Conjugation Reactions}

Functionalization of APTES-containing glass with maleic anhydride (APTES-SG-MA APTS25SG423MA). $0.50 \mathrm{~g}$ of APTS25SG423 APTES-SG glass (1.75 $\left.\mathrm{mmol} \mathrm{NH}_{2}\right), 0.17 \mathrm{~g}$ of maleic anhydride $(1.75 \mathrm{mmol})$ and $10 \mathrm{~mL}$ anhydrous diethyl ether were introduced in a round bottom flask, previously dried in oven and flushed with Ar. The suspension was let under stirring at room temperature for $5 \mathrm{~min}$, then filtered on Buchner funnel and washed with acetone, yielding $0.22 \mathrm{~g}$ of APTES-SG-MA APTS25SG423MA. The amount of $\mathrm{NH}_{2}$ groups available at the glass surface after reaction, determined by acid-basic titration, was $0,9 \mathrm{mmol} / \mathrm{g}$. This datum indicate that the degree of conjugation is $\sim 75 \%$ (See Table 1). 
Conjugation of APTS25SG423MA APTES-SG-MA with 5-aminofluorescein (APTES-SG-MA-AF) APTS25SG423MA AF). $1.47 \mathrm{~g}$ of maleilated APTS25SG423 APTES-SG (3.82 mmol -COOH), 2.93 $\mathrm{g}$ of 1-ethyl-3-(3-dimethylaminopropyl) carbodiimide hydrochloride (15.3 mmol), $1.76 \mathrm{~g} \mathrm{~N}$ hydroxysuccinimide $(15.3 \mathrm{mmol})$ and $110 \mathrm{ml}$ anhydrous DMSO were introduced in a round bottom flask, previously dried in oven and flushed with Ar, and let under stirring for 30 min at r.t. in dark; 5-aminofluorescein $(1.33 \mathrm{~g}, 2.98 \mathrm{mmol})$ was then dissolved in $30 \mathrm{~mL}$ anh. DMSO and flushed with Ar; the orange solution was added dropwise to the glass suspension, and the mixture was let under stirring at r.t. in dark for 1 hour, then filtered on a Buchner funnel and washed with DMSO and acetone, giving $1.35 \mathrm{~g}$ of APTS25SG423MA AF APTES-SG-MA-AF conjugate. The conjugation was confirmed by means of Raman spectroscopy and TGA analysis (See Results and Discussion Section).

Conjugation of APTS25SG423 APTES-SG with N-(5-Fluoresceinyl)maleamic acid (APTES-SGMAAF APTS25SG423 MAAF). N-(5-Fluoresceinyl)maleamic acid (MAAF) was synthesized as described in literature.[32] $2.2 \mathrm{~g}$ of $\mathrm{N}$-(5-fluoresceinyl)maleamic acid (4.93 mmol), $3.78 \mathrm{~g}$ of 1ethyl-3-(3-dimethylaminopropyl) carbodiimide hydrochloride $(19.7 \mathrm{mmol}), \quad 2.27 \mathrm{~g} \quad \mathrm{~N}$ hydroxysuccinimide $(19.7 \mathrm{mmol})$ and $140 \mathrm{ml}$ anhydrous DMSO were introduced in a round bottom flask, previously dried in oven and flushed with Ar, and let under stirring for 3 hours at r.t. in dark, giving a dark yellow solution; $1.41 \mathrm{~g}$ of APTS25SG423 APTES-SG glass (4.93 $\mathrm{mmol} \mathrm{NH}_{2}$ ) were then introduced and the mixture was reacted at r.t. in dark for 1 hour, then filtered on a Buchner funnel and washed with DMSO and acetone, giving $1.31 \mathrm{~g}$ of APTS25SG423 MAAF APTES-SG$M A A F$ conjugate. The conjugation was confirmed by means of Raman spectroscopy and TGA analysis (See Results and Discussion Section).

\section{Characterization techniques}

Scanning electron microscopy (SEM). SEM microscopy was used to investigate any effect of the on the morphology of bioactive glasses after the functionalization/conjugation reactions. The images were obtained with a ZEISS ECO 50 XVP microscope equipped with a LaB6 source, with acquisition of images at $15 \mathrm{kV}$. For each sample, four different sample areas were investigated.

Raman spectroscopy. FT-Raman spectra were collected directly on the glass powders in the 4000$100 \mathrm{~cm}^{-1}$ range. A Bruker Vertex 70 instrument with the RAM II accessory, equipped with NdYAG laser $(1064 \mathrm{~nm})$ was used: 15000 scans with a mean laser power of $50 \mathrm{~mW}$ were accumulated for each sample. 
Acid-base titration. Acid-base titration was conducted by suspending the sample $(50 \mathrm{mg})$ in $10 \mathrm{ml}$ of $\mathrm{HCl}$ solution $(0.1 \mathrm{M})$ for 1 hour. Then, the solution was centrifuged to remove glass powder and $4 \mathrm{ml}$ of the acid solution (after the glass contact) are titrated with $\mathrm{NaOH}$ solution $(0.1 \mathrm{M})$ using phenolphthalein as indicator. Data were a mean of three independent determinations on different samples.

Thermogravimetric analyses and differential thermal calorimetry (TGA-DSC). To determine the amount of organic functionalization, thermo gravimetric analyses coupled with differential thermal calorimetry are performed. A TA instrument Q600 SDT connected with a simultaneous DSC-TGA heat flow analyser has been used.

All measurements are carried out under $\mathrm{N}_{2}$ flow (ramp: $10{ }^{\circ} \mathrm{C} \cdot \mathrm{min}^{-1}$ ) in the $50-900{ }^{\circ} \mathrm{C}$ temperature range.

\section{pH-Dependent release tests}

In order to evaluate the bond stability at different $\mathrm{pH}$ values, $10 \mathrm{mg}$ of conjugate systems were soaked in $2 \mathrm{ml}$ of buffered solution at different times: 4 hours, 2 and 7 days. Two buffered solutions are used: $i$ ) buffer at $\mathrm{pH}=4.2$ (acetic/acetate buffer); ii) buffer at $\mathrm{pH}=7.4$ (PBS buffer). After different soaking times to remove the powder, the solutions were filtrated and analysed by fluorescence spectroscopy.

Fluorescence spectroscopy. All measurements were performed using a JASCO FP6200 fluorescence spectrophotometer. The slits were adjusted to achieve a spectral bandwidth of $2 \mathrm{~nm}$, and the spectra were obtained with a 1-nm step size and 1-s integration time. The samples were excited at a wavelength of $490 \mathrm{~nm}$. The standards of 5-AF were prepared in the concentration range between $10^{-5}-10^{-7} \mathrm{M}$, because in this range the intensity of fluorescence band at $510 \mathrm{~nm}$ was proportional to the concentration (Abs in the range $0.1-0.2$ ). As fluorescence properties of the 5$\mathrm{AF}$ was strictly $\mathrm{pH}$-dependent, before the measurements the $\mathrm{pH}$ of both standard and samples solutions was adjusted to $\mathrm{pH} \sim 7.4$ using phosphate buffered solution (PBS). 


\section{Results and Discussion}

Two different synthetic routes were explored in the conjugation of 5-aminofluorescein to the APTES-containing glass.

In the first approach, the glass was functionalized with maleic anhydride and then 5-AF was conjugated. (Synthesis details are reported in the Methods section)

Please, insert here Scheme 1

In the second approach, 5-AF was reacted to maleic anhydride to give the maleamic acid and then conjugated to the glass. (Synthesis details are reported in the Methods section).

Please, insert here Scheme 2

The second way of synthesis, frequently used in the drug delivery of doxorubicin,[17-23,33,35] particularly fits our system, since glass degradation proved a limiting factor on reaction times. In this synthetic approach, the bioglass has to endure only one reaction step, and is less subject to disgregation on solvents' part.

\section{Samples Characterization}

In Figure 1 SEM micrographs of the samples before and after the functionalization/conjugation reactions are reported.

Please, insert here Figure 1

Concerning the starting glass (Section A) the size of the aggregates is in the 10-50 $\mu \mathrm{m}$ range and its shape is angular.[35] The morphology of the sample after the reaction with maleic anhydride is 
quite similar: it is possible to observe the presence of larger and homogeneously distributed aggregates with respect to the starting glass (probably the anhydride can favour the aggregation; Section B).

In the case of samples conjugated with 5-AF the morphology is modified; the glass aggregates become more regular also with a decreased size. These changes are probably ascribable to the magnetic stirring and to the solvent used in the conjugation reaction. The difference in the conjugation route (first and second route) does not affect the glass morphology.

In Figure 2 Raman spectra carried out on the APTS25SG423 APTES-SG samples before and after the covalent functionalization with maleic anhydride and the conjugation with 5-AF are reported.

Please, insert here Figure 2

By the inspection of Raman spectra the following features can be evidenced:

i) the bands at $\sim 2960, \sim 2940$ and $\sim 2890 \mathrm{~cm}^{-1}$ typical of asymmetric and symmetric C-H stretching vibrations are clearly detectable in all samples; these bands are due to the presence of APTES anhydride and 5-AF molecules. The intensity ratio of these bands changed after the functionalization with the anhydride and 5-AF with respect to APTS25SG423 APTES-SG glass;

ii) the bands at $\sim 1650$ and $\sim 1600 \mathrm{~cm}^{-1}$ are due to carbonyl groups stretching vibrations typical of carboxylic acid and amide, respectively. These bands appear on the glass after the covalent functionalization with the anhydride and 5-AF: the band at $1600 \mathrm{~cm}^{-1}$ is a clear indication of the formation of an amide bond between the $-\mathrm{NH}_{2}$ groups present on the glass surface and the carbonyl group of the anhydride or between the carbonyl group of the anhydride and $-\mathrm{NH}_{2}$ groups of 5-AF; the intensity of this band increased after the conjugation; this is an indication of the formation of the second amide bond. On the opposite, the band at $\sim 1650 \mathrm{~cm}^{-1}$ typical of $\mathrm{C}=\mathrm{O}$ stretching vibration of carboxylic groups resulting from the anhydrides' ring opening decreased after the conjugation with 5-AF, another indication of the successful conjugation reaction.

iii) in the 1450-1200 $\mathrm{cm}^{-1}$ spectral range there are the characteristic bands of $\mathrm{C}-\mathrm{H}$ bending modes;

$i v$ ) the sharp band at $\sim 1040 \mathrm{~cm}^{-1}$ is typical ascribable of both carbonate and silicate species; it can be assigned to both ionic carbonate and to breathing motions of planar three-membered silica rings, $v_{\text {sym }}(\mathrm{Si}-\mathrm{O})$ and $v_{\text {asym }}(\mathrm{Si}-\mathrm{O})$ of the $\mathrm{Si}-\mathrm{O}-\mathrm{Si}$ groups, respectively; indeed, vibrational evidences for 
the presence of Si-O-H groups could also be present in this spectral region. All the mentioned vibrational features fall in the same range and it is not possible to isolate the single bands but the carbonate modes are more intense with respect to silica one.

Raman spectra confirm the efficiency of the covalent functionalization with both anhydride and 5AF with the formation of an amide bond between the anhydride with the amino groups of both glass and 5-AF.

This is a very good result highlighting the ability of functional groups present on the material surface to be conjugated with molecules of biological interest.

In Figure 3 Raman spectra carried out on the APTS25SG423 APTES-SG samples after the conjugation with $\mathrm{N}-(5$-Fluoresceinyl)maleamic acid are reported.

Please, insert here Figure 3

Raman spectrum after glass functionalization with $\mathrm{N}$-(5-Fluoresceinyl)maleamic acid presents the same features described previously confirming the formation of amide bonds. Comparing the spectra of the samples after the two different functionalization routes there are no significative differences.

The amount of 5-AF conjugated with the two different routes are evaluated by means of thermogravimetry (TGA-DSC). Data are reported in Table 2.

Please, insert here Table 2

In order to evaluate the amount of organic molecules present in/on the glass the weight loss in the range between 343-673 $\mathrm{K}$ has been considered. Concerning the starting glass APTS25SG423 APTES-SG the \% of weight loss is $26 \mathrm{wt} \%$ ascribable to the presence of APTES molecules in the glass matrix. After the functionalization with maleic anhydride (APTS25SG423-MA APTES-SGMA) the $\%$ of weight loss is $\sim 27 \mathrm{wt} \%$, this datum is closer to that of the starting glass as a consequence of the low molecular weight of maleic anhydride with respect to glass matrix. After the conjugation reactions with 5-AF the weight loss increased with respect to the starting glass. In 
the case of the first conjugation route (APTS25SG423MA AF APTES-SG-MA-AF), starting from APTS25SG423MA APTES-SG-MA sample, the \% of weight loss is \% $92 \mathrm{wt} \%$ whereas in the case of the second conjugation route (APTS25SG423-MAAF APTES-SG-MAAF), starting from APTS25SG423 APTES-SG sample, the \% of weight loss is $71 \mathrm{wt} \%$. From these data it is possible to conclude that the first method allows to conjugate the greatest amount of 5-AF ( $\approx 65 \mathrm{vs} 44 \mathrm{wt} \%$ ); this difference can be due to the fact that $\mathrm{N}$-(5-Fluoresceinyl)maleamic acid possesses a greater steric hindrance with respect to maleic anhydride preventing the functionalization of vicinal $-\mathrm{NH}_{2}$ glass surface functionalities. As a consequence of the high molecular weight of 5-AF there is an increment of weight losses.

In order to evaluate the efficiency of the $\mathrm{pH}$ sensitive bond after the two conjugation reactions, release tests in buffered solutions at $\mathrm{pH} 7.4$ and 4.2 were performed.

The release data are reported in Figure 4 and in Table 3. The amount of 5-AF released has been determined by means of fluorescence spectroscopy.

Please, insert here Figure 4

The release of 5-AF presents exhibits a time-dependent trend: increasing the soaking times the amount of 5- AF released from both materials functionalization route is increased. This datum confirms the ability of these materials to act as controlled drug delivery system.

Indeed, comparing the release at two different $\mathrm{pH}$ (see Table 3 ) it is possible to note that after one week, at $\mathrm{pH} 4.2$ the amount of 5-AF released at $\mathrm{pH} 4.2$ is higher than at $\mathrm{pH} 7.4$ in both conjugation routes, -The amount of 5 - $\mathrm{AF}$ released is higher at acid $\mathrm{pH}$ with respect to physiological one which is consistent with the development of a pH-sensitive drug delivery system.

This evidence demonstrated that the amount of 5 - $\mathrm{AF}$ released is higher at acid $\mathrm{pH}$ with respect to physiological one which is consistent with the development of a pH-sensitive drug delivery system

Please, insert here Table 3 
For short soaking times, however, in the first conjugation route the amount of 5-AF released at physiological $\mathrm{pH}$ is higher than of the second route. This result can be explained considering that, in addition to covalent grafting through APTES chains, the 5-AF can interact by means of weak interactions with silanols on the bioactive glasses surface mainly for the first functionalization route [36-38]. In order to remove the physisorbed 5-AF repeated rinsing with polar solvents (DMSO, acetone) were performed on bioactive glass after the functionalization, despite this physisorbed 5$\mathrm{AF}$ was not completely removed and due to its higher solubility in physiological buffer it is released in buffered solution at $\mathrm{pH}$ 7.4.

Finally, comparing the amount of 5-AF released in the case of the two conjugation reactions, for the second conjugation route (less efficient with respect to the first one ascribable to less amount of both physisorbed and covalently bonded 5-AF) the percentage of 5-AF released after one week is lower with respect to the material conjugated with the first route. In the second route of functionalization the conjugate is more stable. This seems to confirm that the amount of physisorbed 5-AF plays a significant role in the first conjugation route.

A pictorial representation of the functionalized bioactive glass and its behaviour in different buffered solutions is reported in Scheme 3.

Please, insert here Scheme 3

Concerning the total amount of 5-AF released, the percentage of 5-AF released after 7 days is less than $z 1 \mathrm{wt} \%$. The maximum release amount is $0.073 \mathrm{mg}$ in $5 \mathrm{~mL}$, corresponding to $14.6 \mathrm{mg} / \mathrm{L}$. This datum is consistent with the very low solubility of 5-AF in aqueous medium $(\sim 11 \mathrm{mg} / \mathrm{L}$ at $\mathrm{pH}$ =4).[39] It is worth to note that, the conditions used for the in-vitro release tests are very different from the ones in the biological environment where there is a continuous flow of biological fluids. This feature was not reproduced in our in-vitro release tests where the buffered solution in contact with the systems did not change.

Indeed, the half maximal inhibitory concentration $\left(\mathrm{IC}_{50}\right)$ for doxorubicin, an anticancer drug commonly used for the treatment of osteosarcoma, is in the range between 0.33-1.1 mg/L.[40] Since the drug could be delivered directly in-situ in the tumour site, avoiding the first-pass metabolism, 
the therapeutic effects can be achieved even with the low amount released from our conjugated bioactive glass. Indeed, the release of a small amount of drug avoids the toxic effects typical of the conventional drug administration.

In order to analyze the effects of glass dissolution in the two buffered solutions after 7 days of soaking, the samples have been analyzed by means of SEM. In Figure 5 the micrographs of the samples before and after 7 days of soaking in buffered solution at $\mathrm{pH} 7.4$ are reported. For the sake of brevity, the micrographs of the samples soaked 7 days at more acid $\mathrm{pH}$ are not reported as no significative morphologies differences can be detected with respect to the material soaked at $\mathrm{pH}=$ 7.4.

Please, insert here Figure 5

After 7 days soaking the glasses aggregates were covered by an external layer made up of new particles whose morphology and composition (see EDS spectrum, section C of Figure 5) is ascribable to the formation of an apatite-like phase. 


\section{Conclusions}

The keystone to building new, specific and smart therapies against cancer in the future consists in the exploitation of the intracellular or external changes.

The development of bioceramics containing a stimuli-responsive covalent bond which can be hydrolyzed in acidic medium, is an ever-evolving research field for specific cancer therapies.

The synergy of the bioactive behaviour of bioceramics together with their capability for local drug delivery is an outstanding perspective for bone therapy purposes.

In the present paper we reported preliminary data for the development of a smart $\mathrm{pH}$-responsive bioactive glass.

Bioactive glass has been conjugated with 5-aminofluorescein (5-AF), mimetic for a aminocontaining anticancer drugs such as doxorubicin using a $\mathrm{pH}$-sensitive spacer (maleic anhydride).

The physico-chemical characterization techniques confirm the glass conjugation.

Release tests were performed at two different $\mathrm{pH}$ (4.2 and 7.4) showing a higher release at acid $\mathrm{pH}$ with respect to physiological one. This preliminary datum can be a useful tool in the development of a $\mathrm{pH}$-sensitive drug delivery system.

The percentage of 5-AF released after 7 days is less than $1 \mathrm{wt} \% .2 \%$ Despite the low solubility of 5$\mathrm{AF}$ and considering the very low $\mathrm{IC}_{50}$ for doxorubicin, the therapeutic effects can be achieved even with the low amount released from our system where the drug would be delivered directly in the tumour site.

These preliminary data are promising results in view of the development of a $\mathrm{pH}$-sensitive drug delivery system. As future perspective, since aromatic amines are less basic and less nucleophilic than their alkyl counterparts due to the resonance effect, the aromatic amine of 5-AF will be derivatized in order to introduce an aliphatic one. Indeed, doxorubicin, an anticancer drug containing an amino group in the structure, will be used instead of 5-AF and a complete more detailed release kinetics will be performed. 


\section{Acknowledgements}

The authors acknowledge Compagnia di San Paolo for the financial support (Project: ORTO11RRT5).

G.L. acknowledges Fondazione di Vignola for the financial support (Progetto "Materiali per la teranostica: progettazione di sistemi contenenti nanoparticelle e molecole di interesse biologico").

All authors acknowledge Dr. C. Giovannoli of University of Torino for acid-basic titration measurements, Prof. E. Diana of University of Torino for Raman measurements.

The authors thank Ms. F. Malafronte and Mr. W. Intelisano for their valuable help. 
Schemes

Scheme 1. Synthesis of APTS25SG423MA APTES-SG-MA and conjugation with 5aminofluorescein.

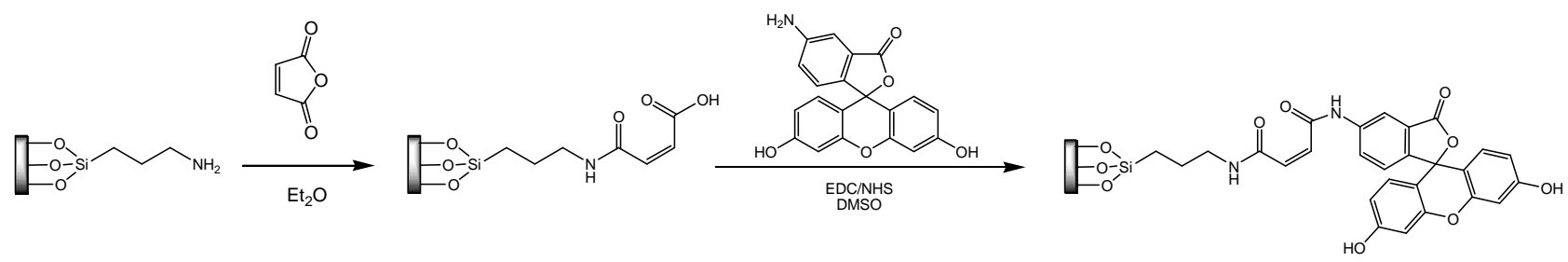

Scheme 2. Synthesis of N-(5-Fluoresceinyl)maleamic acid and conjugation to APTES-SG. APTS25SG423.

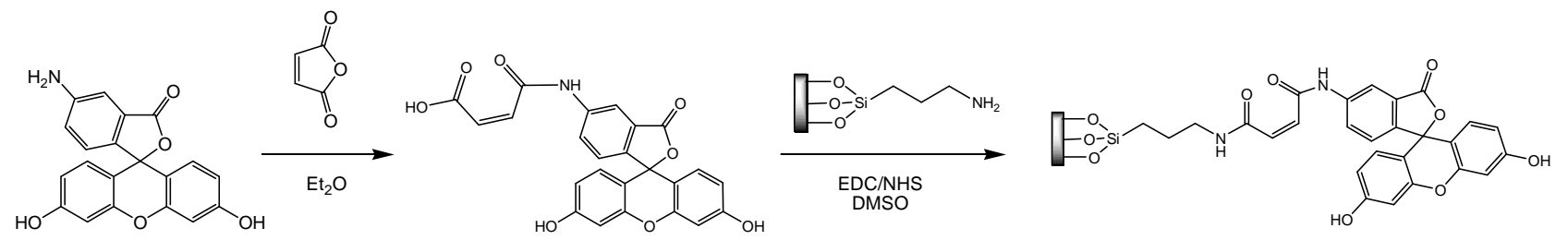

Scheme 3. Representation of the different behaviours of the $\mathrm{pH}$-responsive bioactive glass in buffered solutions at different $\mathrm{pH}$.

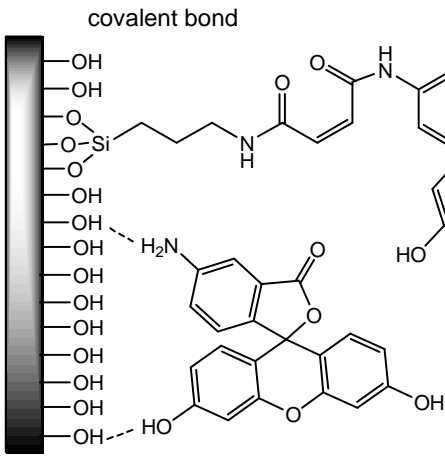

hydrogen bond
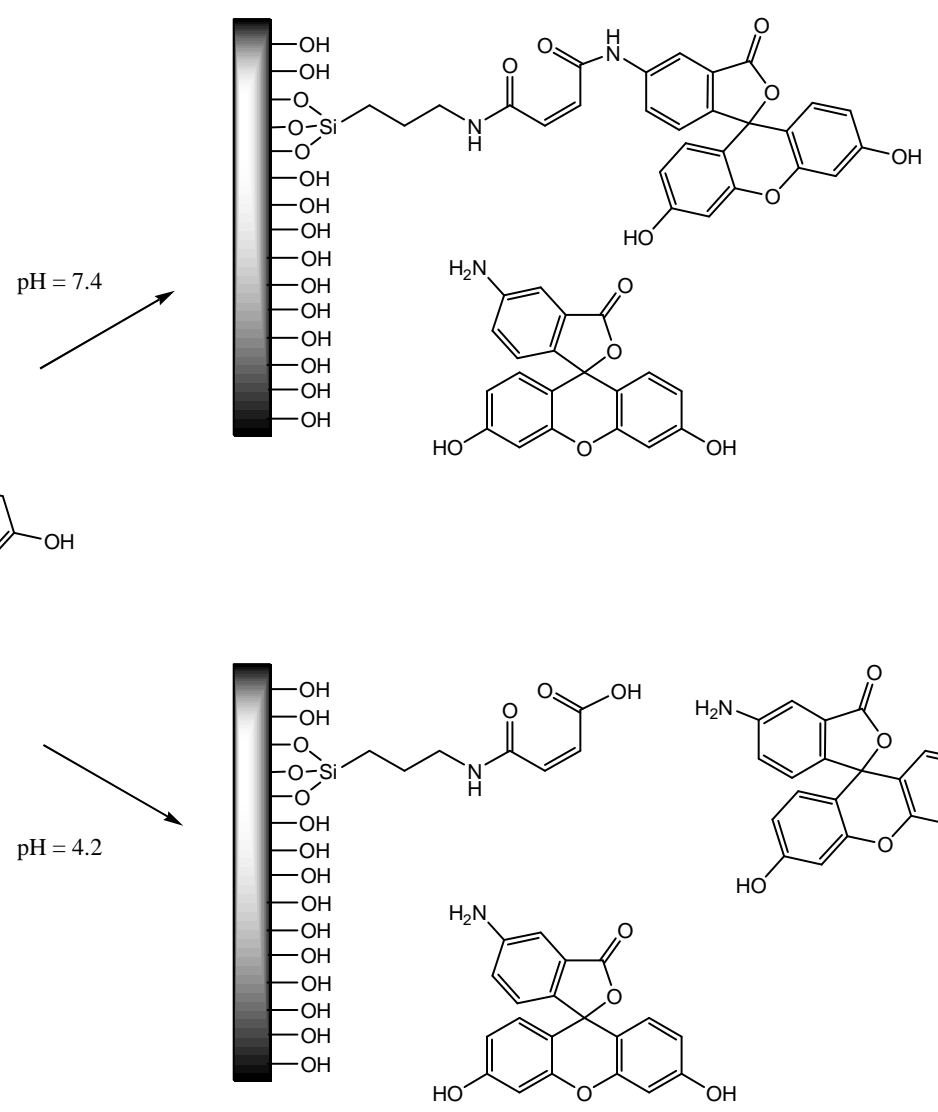


\section{Figures.}

Figure 1. SEM micrographs (500X)of the APTES-glass before and after the conjugation reactions. Section A: as-synthesized glass (APTS25SG423 APTES-SG); Section B: APTS25SG423 APTESSG glass functionalized with maleic anhydride (APTES-SG-MA) ; Section C: APTS25SG423 APTES-SG glass conjugated with maleic anhydride and 5-aminofluorescien (APTES-SG-MA-AF). (5-AF).
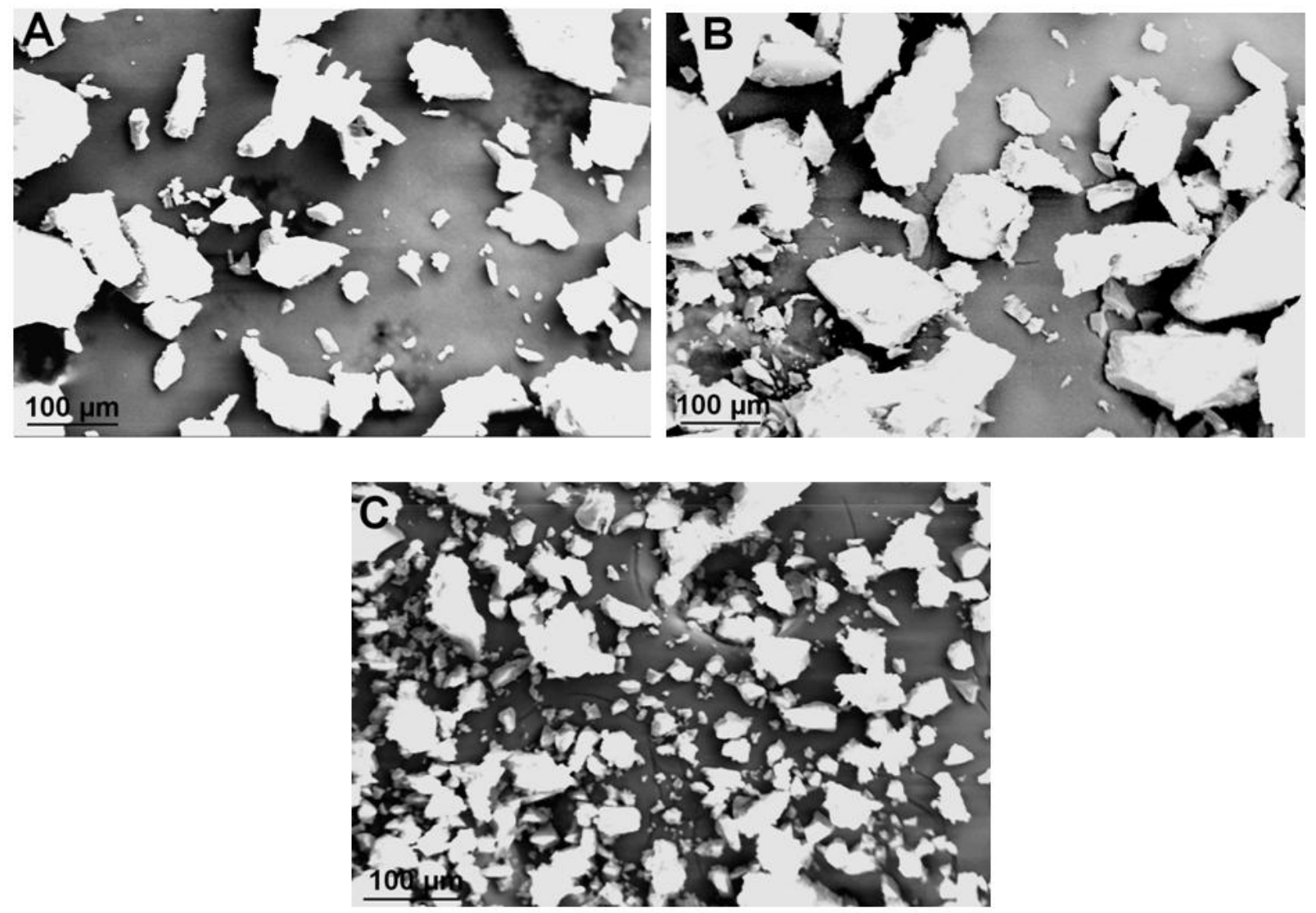
Figure 2. Raman spectra in the $3300-400 \mathrm{~cm}^{-1}$ spectral range carried out on: (a) as-synthetized samples (APTES-SG); (b) APTS25SG423 APTES-SG after functionalization with maleic anhydride (APTS25SG423MA APTES-SG-MA); (c) APTS25SG423MA APTES-SG-MA after functionalization with 5-aminofluorescein (APTES-SG-MA-AF) (c) are reported. As reference, the spectrum of pure 5-aminofluorescein (5-AF) is reported (see spectrum C). Blown-up in the 1700$1500 \mathrm{~cm}^{-1}$ spectral range.
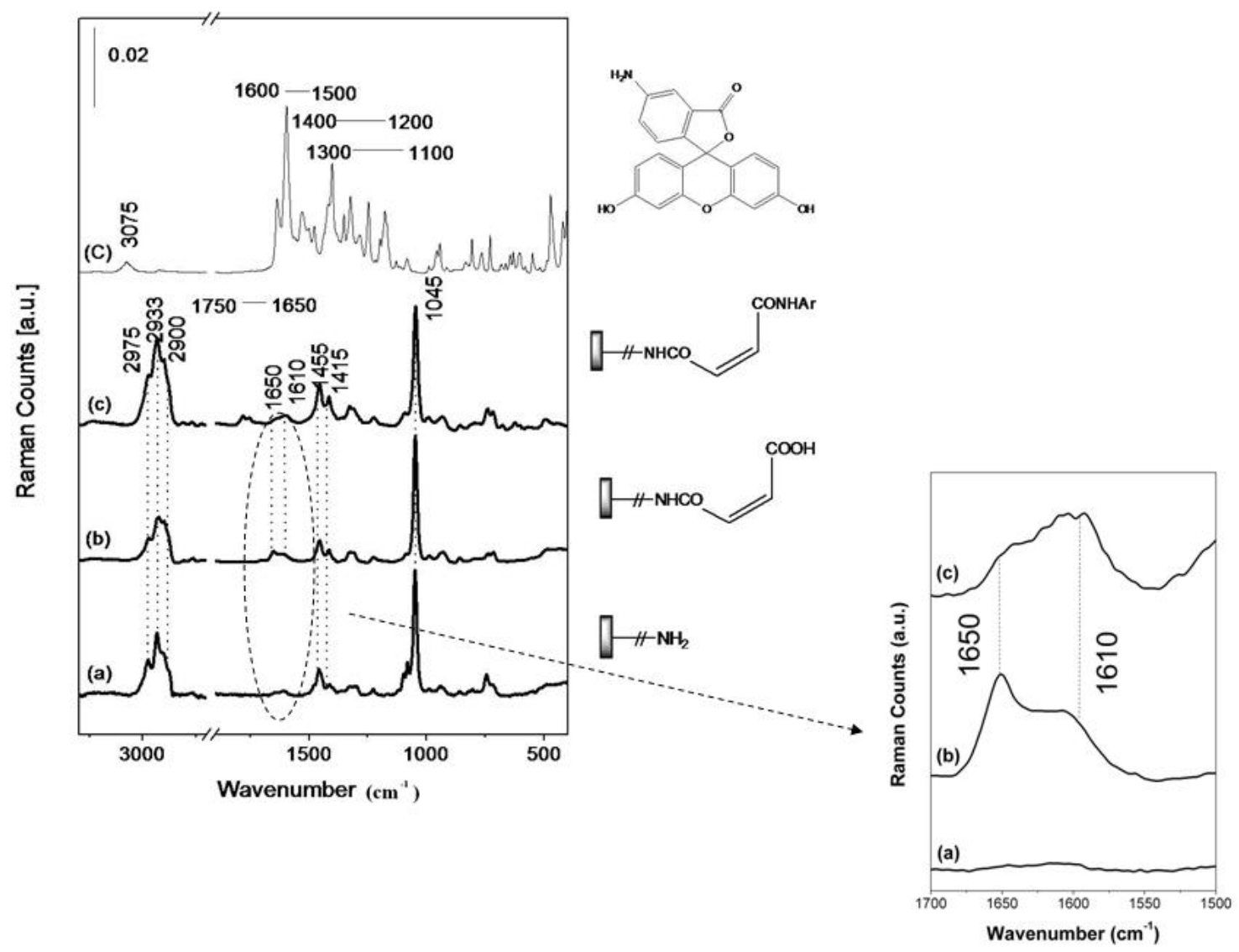
Figure 3. Raman spectra in the $3300-400 \mathrm{~cm}^{-1}$ spectral range carried out on: (a) as-synthetized samples (APTES-SG); (b) APTS25SG423 APTES-SG after functionalization with N-(5Fluoresceinyl)maleamic acid (APTES-SG-MAAF). As reference, the spectrum of N-(5Fluoresceinyl)maleamic acid (MAAF) is reported (see spectrum D). Blown-up in the 1700-1500 $\mathrm{cm}^{-1}$ spectral range.

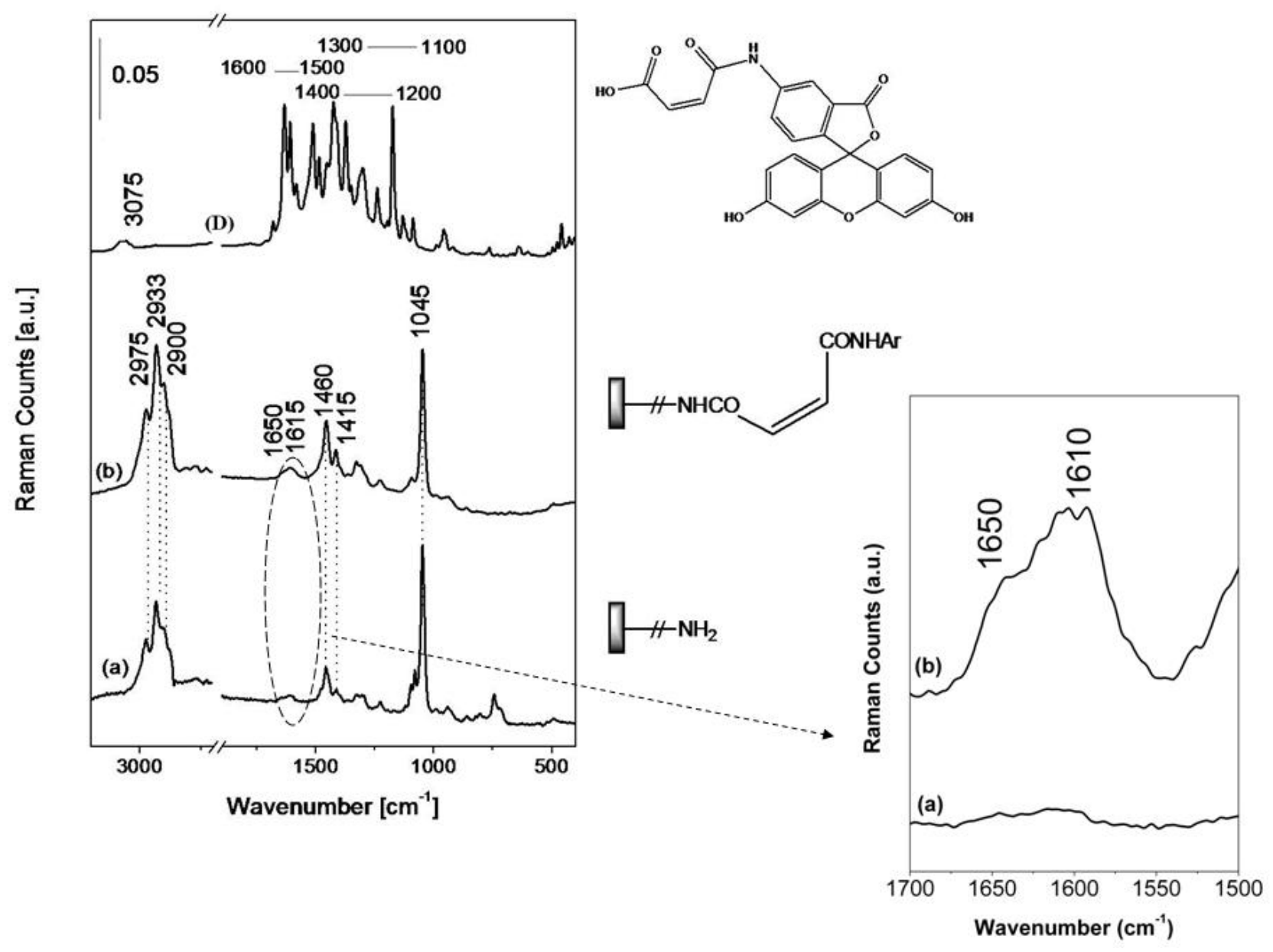


Figure 4- Section A: Release data reported as $\mathrm{mg}$ of 5-aminofluorescein (5-AF) released in $5 \mathrm{~mL}$ of solution at different times (4 hours, 2 and 7 days) at two-different $\mathrm{pH} 4.5$ for both conjugation routes. Section B: Release data reported as \% of 5-aminofluorescein (5-AF) released at different times (4 hours, 2 and 7 days) at two different $\mathrm{pH} 4.5$ for both conjugation routes. Section $A$ : Phosphate buffer solution $\mathrm{pH}=7.4$; Section $B$ Acetic/Acetate buffer solution $\mathrm{pH}=4.2$. Square symbols (-): first conjugation route; Circle symbols (-): second conjugation route; Solid symbols data reported as \% of 5 AF released; open symbols data reported as mg of 5 -AF released.
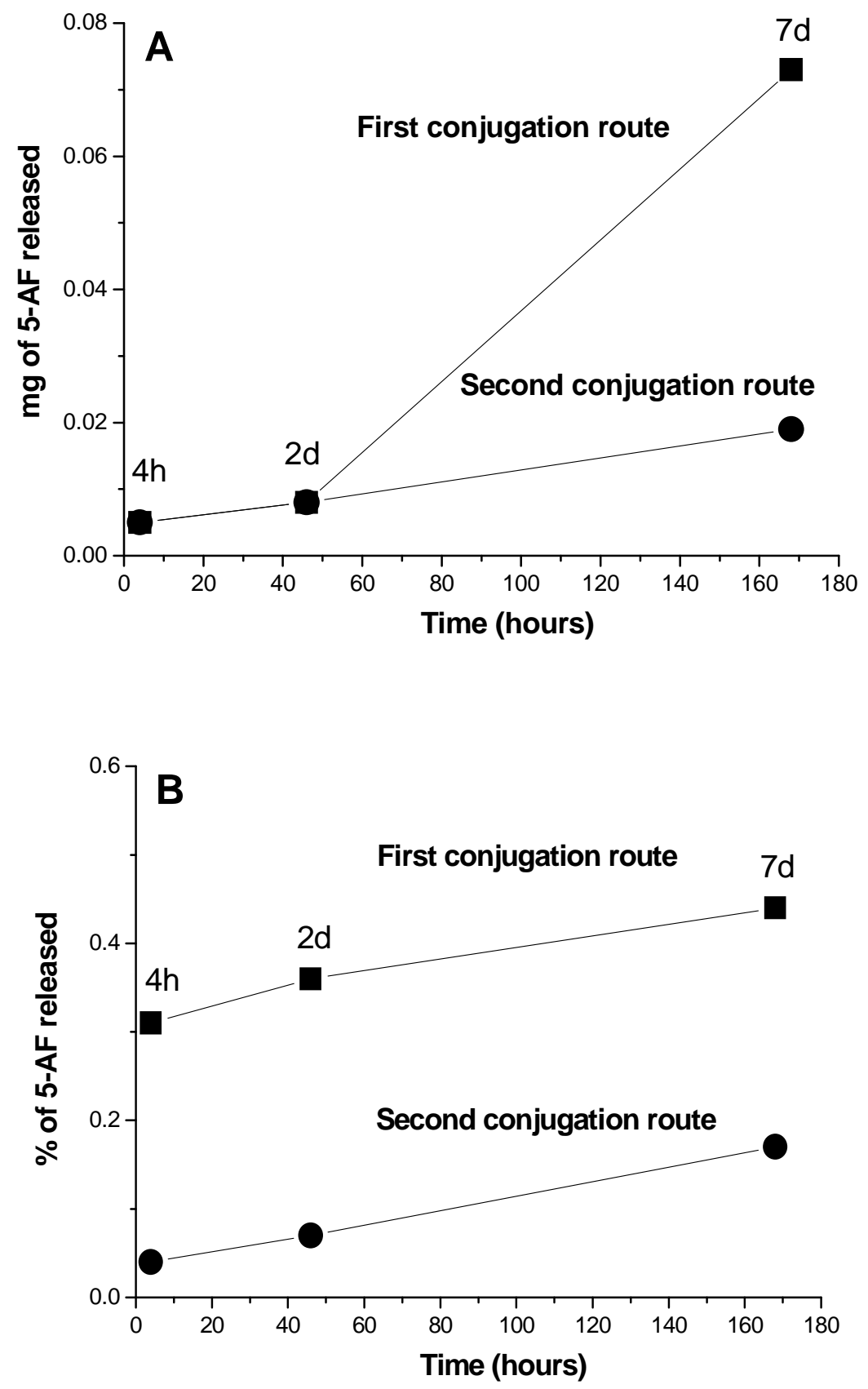
Figure 5. SEM micrographs of the sample APTES-25-SG-MA-AF as synthesized (Section A) and after 7 days of soaking in buffered solution at $\mathrm{pH}$ 7,4 (Section B) are reported. Section C: EDS spectrum.
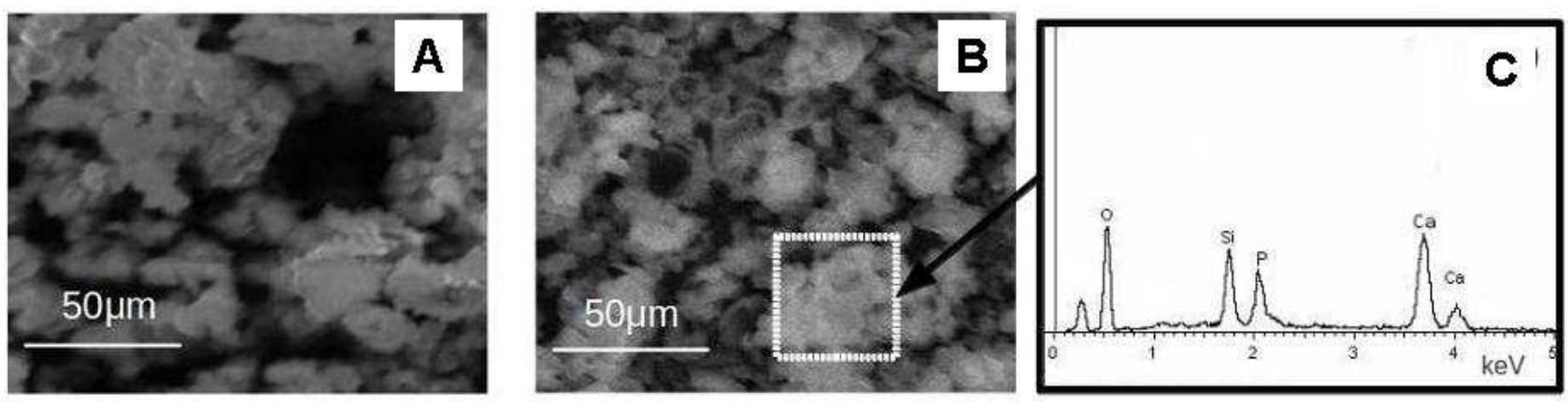
Tables.

Table 1. Acid-base titration: amount of free $-\mathrm{NH}_{2}$ onto the glass surface before and after the functionalization/conjugation reactions.

Samples

APTS25SG423 APTES-SG

APTS25SG423 APTES-SG-MA
Amount of free $-\mathrm{NH}_{2}$

( $\mathrm{mmol} / \mathrm{g}$ )

$3.5 \pm 0.1$

$0.90 \pm 0.1$
$\%$ of glass $-\mathrm{NH}_{2}$

functionalized

I

$74 \%$ 
Table 2. Percentage of weight loss in the temperature range between $373-673 \mathrm{~K}$ for the glass assynthesized and after each functionalization/conjugation reactions. Data are determined by means of TGA analysis.

\section{Samples}

APTS25SG423-APTES-SG

APTS25SG423 APTES-SG-MA

APTS25SG423MA APTES-SG-MA-AF

APTS25SG423 APTES-SG -MAAF
\% Weight loss ( wt \%)

(373-673 K)

$26 \pm 1 \%$

$27 \pm 1 \%$

$92 \pm 1 \%$

$71 \pm 1 \%$ 
Table 3. Amount of 5-AF released in solution at two different $\mathrm{pH}$ (7.4 PBS buffer and $\mathrm{pH} 4.2$ acetic/acetate buffer) after 4 hours, 2 and 7 days soaking time. Data are reported as i) mg of 5-AF released in $5 \mathrm{~mL}$ of buffered solution (starting from $25 \mathrm{mg}$ of samples soaked in $5 \mathrm{~mL}$ ) or ii) percentage of 5-AF released in brackets.

APTS25SG423 APTES-SG-MA-AF $16.25 \mathrm{mg}(65 \%)$ of 5 -AF in $25 \mathrm{mg}$ of glass

mg of 5-AF released

(\% value)
APTS25SG423 APTES-SG-MAAF $11.20 \mathrm{mg}(44 \%)$ of $5-\mathrm{AF}$ in $25 \mathrm{mg}$ of glass

Time

mg of 5-AF released

(\% value)
pH 7.4

$4 \mathrm{~h}$

$2 \mathrm{~d}$

$7 d$

pH $4.2 \quad 4 \mathrm{~h} \quad 0.050 \pm 0.003(0.31 \%) \quad$ pH $4.2 \quad 4 \mathrm{~h} \quad 0.005 \pm 0.001(0.04 \%)$

$2 \mathrm{~d} \quad 0.058 \pm 0.007(0.36 \%)$

$2 \mathrm{~d} \quad 0.008 \pm 0.002(0.07 \%)$

$7 d$

$0.073 \pm 0.009(0.44 \% 1.8$

\%)

$7 \mathrm{~d} \quad 0.019 \pm 0.003(0.17 \%)$

* under the lowest standard (corresponding to $0.005 \mathrm{mg}$ released in $5 \mathrm{ml}$ by $25 \mathrm{mg}$ of sample) 


\section{References}

[1] Gentleman E, Polak, JM. Historic and current strategies in bone tissue engineering: Do we have a hope in Hench? J Mat Sci Mat in Med. 2006;17:1029-35.

[2]. Arcos D, Vallet-Regì M. Bioceramics for drug delivery. Acta Biomat. 2013;61:890-911.

[3] Manzano M, Vallet-Regi M. Revisiting bioceramics: Bone regenerative and local drug delivery systems. Prog in Sol State Chem. 2012;40:17-30.

[4] Hench LL. J Am Ceram Soc. 1971;74: 487-96.

[5]. Li R, Clark AE, Hench LL. J Appl Biomater. 1991;1:231-9.

[6] Vallet-Regi M, Izquierdo-Barba I, Colilla M. Structure and functionalization of mesoporous bioceramics for bone tissue regeneration and local drug delivery. Phil Trans Roy Soc A-Math Phys and Eng Sci 2012;370:1400-21.

[7] Lopez-Noriega A, Arcos D, Vallet-Regi M. Functionalizing Mesoporous Bioglasses for Long-Term Anti-Osteoporotic Drug Delivery. Chem a Europ J. 2010;16:10879-86.

[8] Lamoureux F, Trichet V, Chipoy C, Blanchard F, Gouin F, Redini F. Recent advances in the management of osteosarcoma and forthcoming therapeutic strategies. Exp Rew Antican Ther. 2007; $7: 169-81$.

[9] Langer R, Peppas NA. Advances in biomaterials, drug delivery, and bionanotechnology. Aiche J. 2003;49:2990-3006.

[10] McCoy CP, Brady C, Cowley JF, McGlinchey SM, McGoldrick N, Kinnear DJ, Andrews G P, Jones DS. Triggered drug delivery from biomaterials. Exp Op on Drug Del 2010;7:605-16.

[11] Vallet-Regì M. Revisiting ceramics for medical applications. Dalton Trans. 2006;44:521120.

[12]. Verne E, Vitale-Brovarone C, Bui E, Bianchi CL, Boccaccini AR. Surface functionalization of bioactive glasses. J of Biomed Mat Res Part A 2009;90A:981-92.

[13] Contessotto L, Ghedini E, Pinna F, Signoretto M, Cerrato G, Crocella V. Hybrid OrganicInorganic Silica Gel Carriers with Controlled Drug-Delivery Properties. Chem A Europ J. 2011:15 12043-9.

[14]. Arean C, Vesga M, Parra J, Delgado M. Effect of amine and carboxyl functionalization of sub-micrometric MCM-41 spheres on controlled release of cisplatin. Cer Intern. 2013;39:7407-14. [15] Aina V, Marchis T, Cerrato G, Laurenti E, Morterra C, Bergandi L, Ghigo D, Lusvardi G, Malavasi G, Menabue L. Novel bio-conjugate materials to decrease the oxidative stress in in-vitro cellular tests: soybean peroxidase immobilized on bioactive glasses. J Mat Chem. 2011;21:1097081. 
[16] Nichele V, Signoretto M, Ghedini E. beta-Galactosidase entrapment in silica gel matrices for a more effective treatment of lactose intolerance. J of Mol Cat B-Enz. 2011;139:189-196.

[17] Ulbrich K, Etrych T, Chytil P, Jelinkova M, Rihova B. HPMA copolymers with pHcontrolled release of doxorubicin - In vitro cytotoxicity and in vivo antitumor activity. J Contr Rel 2003;87:33-47.

[18] Shen WC, Ryser HJ. cis-Aconityl spacer between daunomycin and macromolecular carriers: a model of $\mathrm{pH}$-sensitive linkage releasing drug from a lysosomotropic conjugate. Biochem and Biophys Res Comm. 1981;102:1048-54.

[19] Manchun S, Dass CR, Sriamornsak P. Targeted therapy for cancer using pH-responsive nanocarrier systems. Life Science 2012;90: 381-87.

[20] Ulbrich K, Subr V. Polymeric anticancer drugs with pH-controlled activation. Adv Drug Del Rev 2004;56:1023-50.

[21]. Lavignac N, Nicholls JL, Ferruti P, Duncan R. Poly(amidoamine) Conjugates Containing Doxorubicin Bound via an Acid-Sensitive Linker. Macromol Biosci 2009;9:480-7.

[22] Ulbrich K, Subr V. Polymeric anticancer drugs with pH-controlled activation. Adv Drug Del Rev. 2004;56:1023-50.

[23]. Hu F, Liu L, Du Y, Yuan H. Synthesis and antitumor activity of doxorubicin conjugated stearic acid-g-chitosan oligosaccharide polymeric micelles. Biomaterials. 2009;30:6955-63.

[24] Son Y, Jang J, Cho Y, Chung H, Park R, Kwon I, Kim I, Park J, Seo S, Park C, Jeong S. Biodistribution and anti-tumor efficacy of doxorubicin loaded glycol-chitosan nanoaggregates by EPR effect. J Cont Rel.2003;91:135-45.

[25] Yoo SY, Lee TL, Yoon JY, Lee IL, Kim MK, Lee HL. Interfacial adhesion reaction of polyethylene and starch blends using maleated polyethylene reactive compatibilizer. J Appl Pol Sci 2002;83:767-76.

[26] Zhang H, Li F, Yi J, Cu C, Fan L, Qiao Y, Tao Y, Cheng C, Wu H. Folate-decorated maleilated pullulan-doxorubicin conjugate for active tumor-targeted drug delivery. Europ J Pharm Sci. 2011;42:517-26.

[27]. Kazarian A, Hilder E, Breadmore M. Utilisation of pH stacking in conjunction with a highly absorbing chromophore, 5-aminofluorescein, to improve the sensitivity of capillary electrophoresis for carbohydrate analysis. J Chromatog A. 2008;1200:84-91.

[28] Boga C, Puggioli S, Gherpelli M, Farruggia G, Pagnotta E, Masotti L, Neyroz P. Fluorescein conjugates of 9-and 10-hydroxystearic acids: synthetic strategies, photophysical characterization, and confocal microscopy applications. Anal Biochem 2004;335:196-209. 
[29] Miletto I, Gilardino A, Zamburlin P, Dalmazzo S, Lovisolo D, Caputo G, Viscardi G, Martra, G. Highly bright and photostable cyanine dye-doped silica nanoparticles for optical imaging: photophysical characterization and cell tests. Dyes and Pigm. 2010;84:121-7.

[30]. Barbero N, Barni E, Barolo C, Quagliotto P, Viscardi G, Napione L, Pavan S, Bussolino F. A study of the interaction between fluorescein sodium salt and bovine serum albumin by steadystate fluorescence. Dyes and Pigm. 2009;80:307-13.

[31] Alberto G, Miletto I, Viscardi G, Caputo G, Latterini L, Coluccia S, Martra G. Hybrid cyanine-silica nanoparticles: homogeneous photoemission behavior of entrapped fluorophores and consequent high brightness enhancement. J Phys Chem C. 2009;113:21048-53.

[32] Reddy P, Kondo S, Fujita S, Toru T. Efficient synthesis of fluorophore-linked maleimide derivatives. Synth-Stutt 1998;7:999-1002.

[33] Remenyi J, Balazs B, Toth S, Falus A, Toth G, Hudecz F. Isomer-dependent daunomycin release and in vitro antitumour effect of cis-aconityl-daunomycin. Biochem Biophys Res Comm. 2003;303:556-61.

[34] Kakinoki A, Kaneo Y, Ikeda Y, Tanaka T, Fujita K. Synthesis of poly(vinyl alcohol)doxorubicin conjugates containing cis-aconityl acid-cleavable bond and its isomer dependent doxorubicin release. Biol and Pharm Bull 2008;31:103-10.

[35] Lusvardi G, Malavasi G, Menabue L, Shruti S. Gallium-containing phosphosilicate glasses: Functionalization and in-vitro bioactivity. Materials Science and Engineering: C, 2013;33:3190-6.

[36] Dugas V, Elaissari A, Chevalier Y. Surface sensitization techniques and recognition receptors immobilization on biosensors and microarrays. Recog. Recep. in Biosensor, Ed. M. Zourob, New York, Springer, 2010;47:134.

[37] Asenath SE, Che W. How to prevent the loss of surface functionality derived from aminosilanes. Langmuir, 2008;24:12405-9.

[38] Russo L, Taraballi F, Lupo C, Poveda A, Jimenez-Barbero J, Sandri M, Tampieri A, Nicotra F, Cipolla R. Carbonate hydroxyapatite functionalization: a comparative study towards (bio)molecules fixation. Interf. Focus, 2014;4:20130040-65.

[39] Calculated using Advanced Chemistry development (ACD/Labs) Software V11.02 (c 19942013 ACD/Labs)

[40] Ray-Coquard I, Le Cesne A. A role for maintenance therapy in managing sarcoma. Canc Treat Rev 2012;35:368-78. 
